\title{
Embryonic Nervous System
}

National Cancer Institute

\section{Source}

National Cancer Institute. Embryonic Nervous System. NCI Thesaurus. Code C34156.

The development of both the central and peripheral nervous systems, which begins during the third week of gestation. 